\title{
Intelligent Battery Management Design and Realization of Emergency Lights Base on Time Division Multiplexing and Balanced Charging Technology
}

\author{
Chunyan $\mathrm{Xu}^{1}$, Feng Jiang ${ }^{2}$, Yunning $\mathrm{Zhao}^{3}$ and Tao $\mathrm{Xu}^{4}$ \\ ${ }^{1,2,4}$ College of Computer Science and Technology, Beihua University, Jilin, \\ 132021, China; \\ ${ }^{3}$ School of Management and Economics, China University of Geosciences, Wuhan, \\ 430074, China \\ xuchunyanjsj@163.com
}

\begin{abstract}
This paper introduces a design and realization method of battery management system about emergency light based on time division multiplexing and balanced charging technology. Through Calculating state of electric charge by detecting the battery charge and discharge current and monitoring of a single voltage, the system achieves a balanced charge control methods and strategies, avoided battery overcharge and over-discharge, and further improves the efficiency of the battery, extending the battery life.
\end{abstract}

Keywords: Emergency lights; Time division multiplexing; Balanced charging; BMS

\section{Introduction}

Now, with lithium-ion batteries, nickel metal hydride batteries, nickel-zinc battery applications, security, reliability, service life and efficiency of spare battery pack in the emergency lamps have a critical impact on the performance of emergency lights [1]. Currently, emergency lights deficiencies are following several aspects, the battery of emergency lights is charging, shelved and uncertainty discharge cycle work state for Long-term, the single battery life and stability of power supply is affected relatively large ,Emergency lighting products, generally only work in emergency mode [2], it starts work when $220 \mathrm{~V}$ AC power supply is stopped. Due to the single mode, emergency lights are more restricted applications range, In the battery pack constituted by single cell in series, Performance of the battery pack is determined by the single cells, especially with the increasing in the charge and discharge cycles, the deviation of each single battery performance consistency also increased [3], affected the normal work of emergency lights; Universal charging control unit of emergency lights are often used non-intelligent acquisition and control, control methods are a single. in order to solve the above problems, emergency light battery management system designed in this paper mainly uses time division multiplexing (DTM) [4] and equalization techniques to effectively monitor the battery pack, charge and discharge management and balance control, to avoid the battery overcharged and over-discharge, to reduce or minimize the deviation of each single cell performance consistency and reduce battery performance decay rate, and further extend battery life, meet the requirements of human-computer interaction and multi-mode switching, enhanced operability and expanded emergency lights range of applications. 


\section{Hardware Design}

\subsection{Hardware Overall Design}

Intelligent emergency lights battery management system (battery management system: BMS) hardware design consists mainly of embedded microcontrollers, memory unit, SMBus bus interface unit, battery voltage and current data acquisition unit, battery status monitoring unit, work status display unit, remote control unit, charge control unit and Low-voltage automatic power unit, LED work lights and fans etc. Each hardware unit attached to the SMBus I/O bus, where some modules via the SMBus bus expansion port, connected to SMBus, which is in favor of the circuit using modular. To comply with emergency lights intelligent industrial design requirements, the specific equipment adopts nickel-zinc D-type battery, nominal capacity 6.50Ah [5]. The hardware diagram of emergency light is shown in Figure 1.

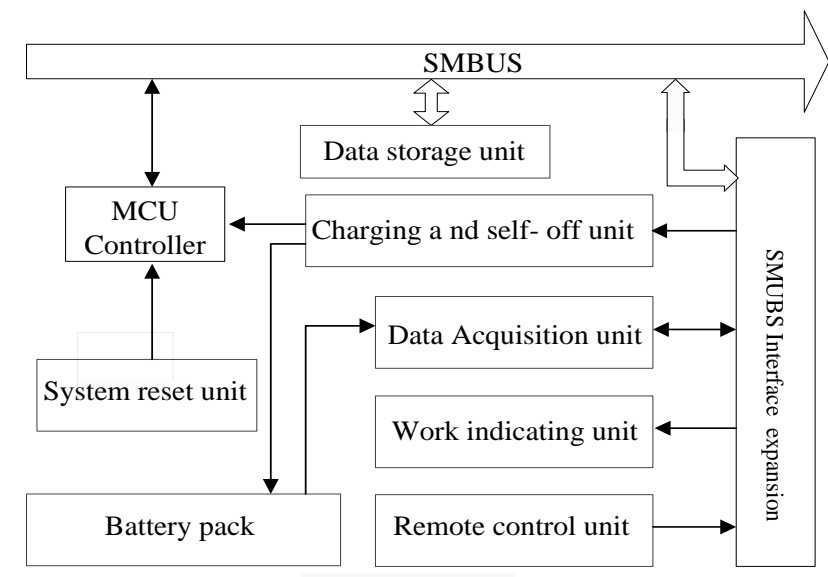

Figure 1.The Function Unit Composition Diagram of Emergency Light

\subsection{Microprocessor}

Using a low-power mixed-signal system-type MCU as microprocessor chip, C8051F410 of Silicon Labs company which uses CIP-51 microcontroller core of Silicon Labs' patented [6]. CIP-51 and MCS-51TM instruction set is fully compatible, which includes 256 bytes of data RAM, program memory contains 32KB FLASH. High-speed 8051 microcontroller core speeds up to 50MIPS (clock frequency of 50MHz), $12 \mathrm{ADC}$, built-in temperature sensor, two 12 current output DAC, can be used simultaneously SMBus ( $\mathrm{I}^{2} \mathrm{C}$ compatible), SPI and UART serial ports.C8051F410 device has four operating modes: Active (normal), idle, suspend and shutdown. When the oscillator and peripherals are active, MCU works in the normal way in idle mode, CPU stops running, and peripherals and internal clocks are active. In suspend mode, SYSCLK stop until there is a wake event occurred, all peripherals are stopped [7]. In shutdown mode, CPU stops running, all interrupts and timers are inactive, the internal oscillator is stopped. C8051F410 MCU family has several key improvements in the CIP-51 core and peripheral, C8051F410 MCU family has the CIP-51 core and peripherals to several key improvements to enhance the overall performance and ease of use in end applications. Extended interrupt system allows a large number of analog and digital peripherals to work independently of the microcontroller, the microcontroller is interrupted only when necessary. An interrupt-driven system requires less intervention by the MCU, which has a higher efficiency and achieve multi-tasking real-time systems easier.

The system uses ADC, I2C and external IO interface low-power CPU controller with I2C iron extended memory for storing parameters. By IO interface expansion and 
decoding for relay strobe of cell voltage measuring relays and sampling [8]; Internal two-way ADC of CPU completed the total current and cell voltage measurements. System completes the switch control, AC power detection, infrared remote control interface, chargers and LED indicator control through other IO interface. Minimum control system of C8051F410 microcontroller and peripheral units diagram are shown in Figure 2.

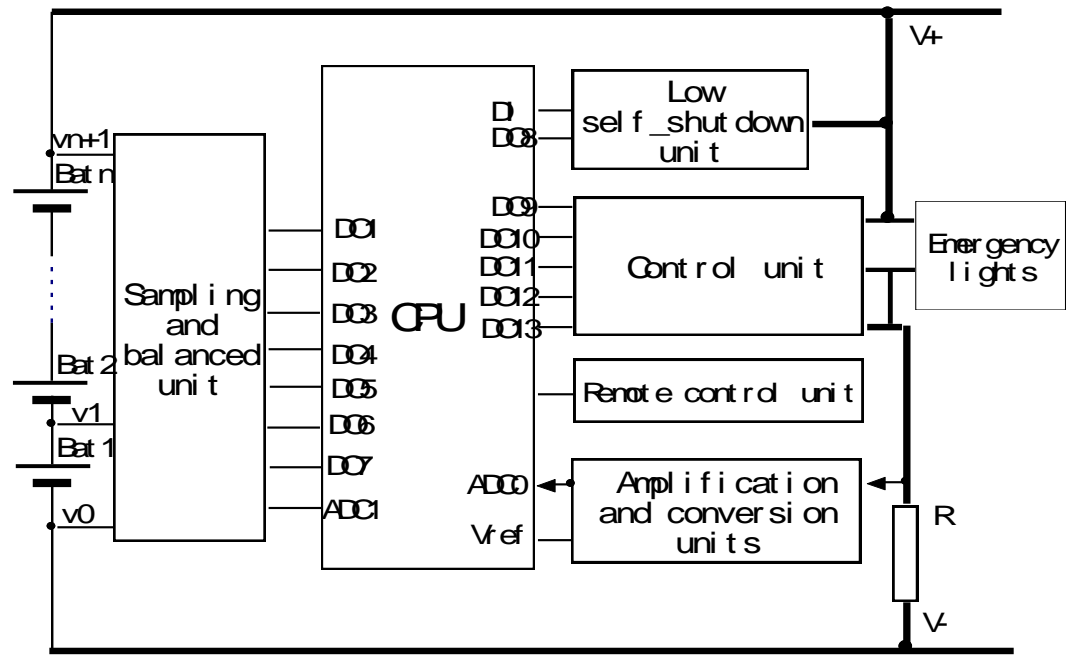

Figure 2. The Minimum Control System of C8051F410

\subsection{SMBus I / O bus}

System Management Bus (System Management Bus: SMBus) is a single ended two wire bus for lightweight information exchange, the most common usage is that the host computer passes instructions to the slave device each other. SMBus I / O bus derives from I2C for low-bandwidth devices on the computer's motherboard, such as computer CPU and subsystem of rechargeable battery, temperature sensors, fan, voltage sensor, Clock chip connection. Card PCI devices can also be connected to SMBus. SMBus is compliant with the System Management Bus Specification Version 2.0 [9], compatible with the I2C serial bus. The system controller reading and writing operations are in bytes, the serial transfer automatically controlled by the SMBus interface data. As a master or slave, the maximum data transfer rate is up to one tenth the system clock frequency. SMBus can operate in master or slave mode, one bus can have multiple master devices. SMBus provides SDA (serial data) control, SCL (serial clock) generation and synchronization, arbitration logic as well as start / stop control and generating circuit. There are three special function registers associated with the SMBus: SMB0CF configuration SMBus, SMB0CN control SMBus state, SMB0DAT data register, used to send and receive data. The operating voltage of SMBus interface can be $3.0 \mathrm{~V}$ and $5.0 \mathrm{~V}$, the operating voltage of different devices on the bus may be different. SCL (serial clock) and SDA (serial data) line is bidirectional, must be pulled through a resistor or similar circuit connected to the power supply voltage. SCL and SDA of Connected to the bus each device must be open-drain or open-collector, so when the bus is free, both lines are pulled high. The maximum number of devices on the bus is limited only by the provisions of the rise and fall times, which are no more than 300ns and 1000ns [10].

All data transmission is started by the master device, you can address one or more the target slave. Master generates a start condition, and then sends the address and direction bit. If this data is writing operation from the master device to the device, after the master sends a data byte, waiting from confirmation from the slave device [11]. If it is a read operation, by sending data from the slave device and waiting for confirmation from 
master device. At the end of the data transfer, the master generates a stop condition to terminate the transaction and release the bus.

\subsection{Current Measurement}

Charge and discharge currents measured by current sampling resistor, realized the measurement of the discharge current at a single power supply, MV level signal current sampling output resistor is amplified to send ADC conversion.

\subsection{Single Voltage Measuring and Balance Control}

Sampling circuit is using the N-way bus switching relays connected in parallel on the voltage measurement as the sampling relay for the completion of each single voltage measurement channel gating, using Parallel capacitance of measured voltage bus completes to measured single voltage maintained, By measuring the voltage on capacitor to realize the roving measurement of each single battery; With balance of optical relay in parallel voltage measurement bus, according to the intelligent balancing algorithm in a balanced cycle, By turning on a single battery discharge circuit, which is composed of single battery relay and balanced sampling relay to achieve the single battery equalization; the sampling equalization unit is consists of Voltage sampling by relays, capacitors and CPU components. As shown in Figure 3.

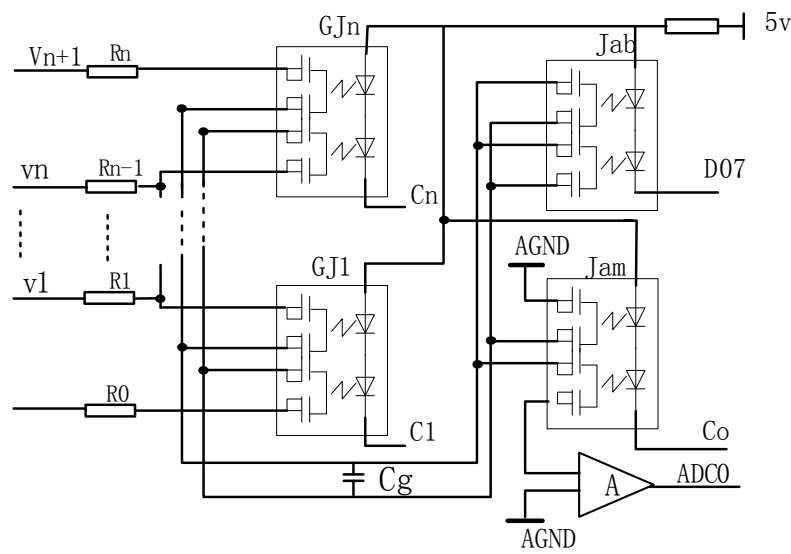

Figure 3. Schematics of Sampling and Equalization Unit

Voltage sampling relay (MOS relay) Gig1 + , Gig2- $(I=1 . . n)$ are connected to the single battery Bati positive and negative, Ji1- connected to one end of the capacitor C, Gig2 + connected to the other end of the capacitor $\mathrm{Cg}$. Gig1, Gig2 closed in turn by $\mathrm{Ci}$ is controlled by software, Bati (the single voltage ) in turn is shifted to capacitors, then In turn connected with the single voltage by Jam to sampling amplifier, amplified single voltage is converted by ADC and saved. Single active equalization of the battery pack is composed of single equalization relay and equalization control relay (Jab), Sampling and equalization are time-sharing work, the single sampling relay Gig acts as a sampling relay while voltage sampling slot, and acts as a equalization relay while time slot in the equalizer, Jab1 + and Jubal + of balance control relay are connected to Gig1- and Gig2 + respectively, so as to constitute a single body equalization circuit. with single voltage sampling measurement bus of $\mathrm{N}$ corresponding to capacitor, in parallel with a equalization relay GJB, When the CPU sequentially sampling and measuring on the single battery voltage, equalization relay GJB in the off state; When the battery is charging, a balanced strobe GJB and the single battery voltage sampling relay Gig which required shunt balanced $i$-th monomers corresponding to constituted the charging bypass circuit, Thereby reducing the battery charge current only to realize shunt balancing. 
Low-power embedded microcontroller core, on the basis of using $\mathrm{N}$ corresponding to 1 single capacitance voltage detection technology, In parallel on a single bus voltage measurement voltage relays and balanced sampling relays, using time division multiplexing method for bus voltage measurement and control ${ }^{[12]}$, achieved a single voltage measurement of $\mathrm{N}$ and balanced charging; Battery charges and discharges current detection based on state of charge (SOC) calculation method with a combination of battery voltage monitoring charge and discharge management, balance control methods and strategies to avoid battery overcharge and over discharge. Specific procedure is as follows.

(1) the system is reset, the microcontroller to keep the ports low and strobe decoder is not any channel, Gig, Jam, Jab are in the off state, so that the single cell measuring counter $\mathrm{i}=0$;

(2) controlled by the CPU samples single voltage the itch relay Gig is turned on and the battery I itch by Gig in parallel measurement sample bus hold capacitor $\mathrm{Cg}$ charged, and after an appropriate time delay, turn-off Gig, in this case the itch battery voltage $\mathrm{Vi}$ is only held on the capacitor $\mathrm{Cg}$;

(3) controlled by the CPU Jam conduced, start ADC, Vs. voltage across Cg is amplified by the amplifier $\mathrm{A} 1$, then the $\mathrm{AD}$ conversion is completed, the sample Vs. values saved, then Jam of controlled by the CPU off;

(4) $\mathrm{I}=\mathrm{I}+1$, if I $<\mathrm{N}$, turn (2), only the cell voltage under measurement, otherwise turn to the next step;

(5) If the battery is in charging state, according to the measured battery voltage and the control algorithm to determine the equalization required for a balanced single battery, Assuming the battery need to be balanced is the $\mathrm{j}$ battery ;

(6) Controlled by the CPU, GJj and Jab turn simultaneously, so that the balanced shunt circuit of the $\mathrm{j}$ single battery is closed and $\mathrm{j}$ single battery is charged shunt equalization.

(7) If the equilibrium cycle timing yet to come, then wait; otherwise controlled by the CPU while GJj and Jab off, go to (2);

(8) End

\subsection{Detection and Control Methods Device of Powered}

External power-off and low battery voltage is detected from the turn-off unit, which comprises a detection relay, AC / DC and external power supply detection circuit composed of GJfback ; GJctr1, GJctr2 and Jwi relays composed of low battery voltage self -shutdown circuit.

(1) Microcontroller reads the output status of GJfback relay, if the state is 1, the current status is the loss of power.

(2) The microcontroller judges whether the battery voltage is not less than UI, if less than 10 , then if less than, then (3)

(3) Micro-controller sends instruction to shut down GJctr2, GJctr2 deadline, due to external power failure, GJctr1 also shut down, at this time Jig opened, the internal system is powered down, thus completing the self turn-off.

(4) End

Adopted by the capacitor step-down AC / DC output low voltage DC signal simultaneously drive two opt couplers GJctr1, GJfback, In which, GJctr1 AC output provides the power supply detection signal of CPU, GJfback provides a driving signal for the power of MOS switch. GJctr2 and GJctr1 are connected in parallel to implement the device of CPU control self-power-off function. Using intelligent powering and self Shutdown control method based on status detection of $220 \mathrm{~V}$ AC power supply and discharge cut-off voltage detection of a single battery, in 220V AC power failure and the 
battery power is exhausted, turned off the power supply of battery management system, to avoid performance degradation or damage caused by battery discharge, but can be restored after the exchanges in the $220 \mathrm{~V}$ power supply and restore power to the battery management control device, but after the $220 \mathrm{~V}$ AC power is restored, the battery management system restore power, Specific schematic diagram is shown in Figure 4.

(1) Micro-controller reads GJfback relay output status, if the state is 1 , then the current power-down state.

(2) the micro-the controller judges whether the battery voltage is less than UI, If greater than or equal, then (4), if less than (3)

(3) Microcontroller closes GJctr2, GJctr2 deadline, due to external power loss, Also GJctr1 in the off state, Jig open this case Jig is opened, the internal system is powered down, thus completing the self turn-off.

(4) End

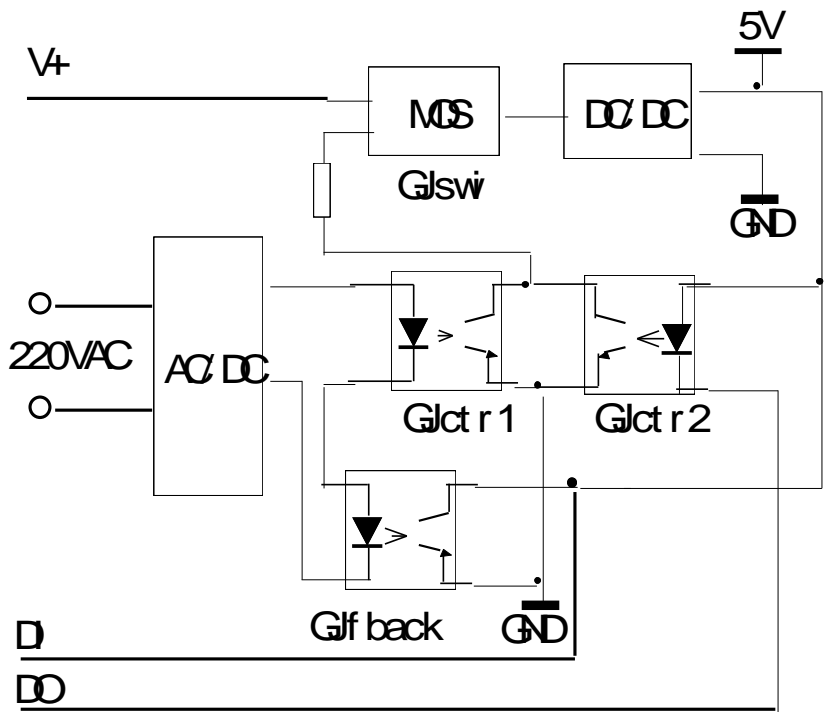

Figure 4. Low Voltage Self-turn Off Schematics

\subsection{IR Remote Control}

To meet the needs of different user applications, emergency lights designed three operating modes, that is idle mode, emergency mode and backup mode, And the use of infrared remote control mode to switch operating modes as well as self-test and activated of batteries, and control emergency lights switch and other functions, For the use of emergency lights and maintenance provides convenient and flexible modes of operation [13]. Using the infrared remote receiver with the CPU interface directly can be realized the remote control function of this device. Three modes of emergency lights switching are operated by the remote controller and the state of $220 \mathrm{AC}$ power, when the value of any one state among the single battery voltage, SOC and the total voltage is below the threshold, the battery is charged until the SOC is $100 \%$, charging cut-off, Power mode switching unit by Jed and GJCharge composed by microprocessor-controlled mode switching.

(1) When Jed and GJCharge are in the open state, emergency lights is off and the charger is not charging for the battery, this state occurs in the external power supply and the battery is fully charged or external power failure and emergency lights is off .

(2) Jed open, when GJCharge closed, emergency light is off and the battery is charging, this state occurs in the external power supply and battery charging, emergency lights off situation [14]. 
(3) Jed closed, when GJCharge opened, emergency lights and powered by a rechargeable machine, this state occurs in the battery is fully charged and the external power supply and requires emergency light on.

(4) Jed and GJCharge are closed, emergency light is on, battery charging, this state occurs in the external power supply, emergency lights, and the battery is not fully charged Work conversions are shown Figure 4.

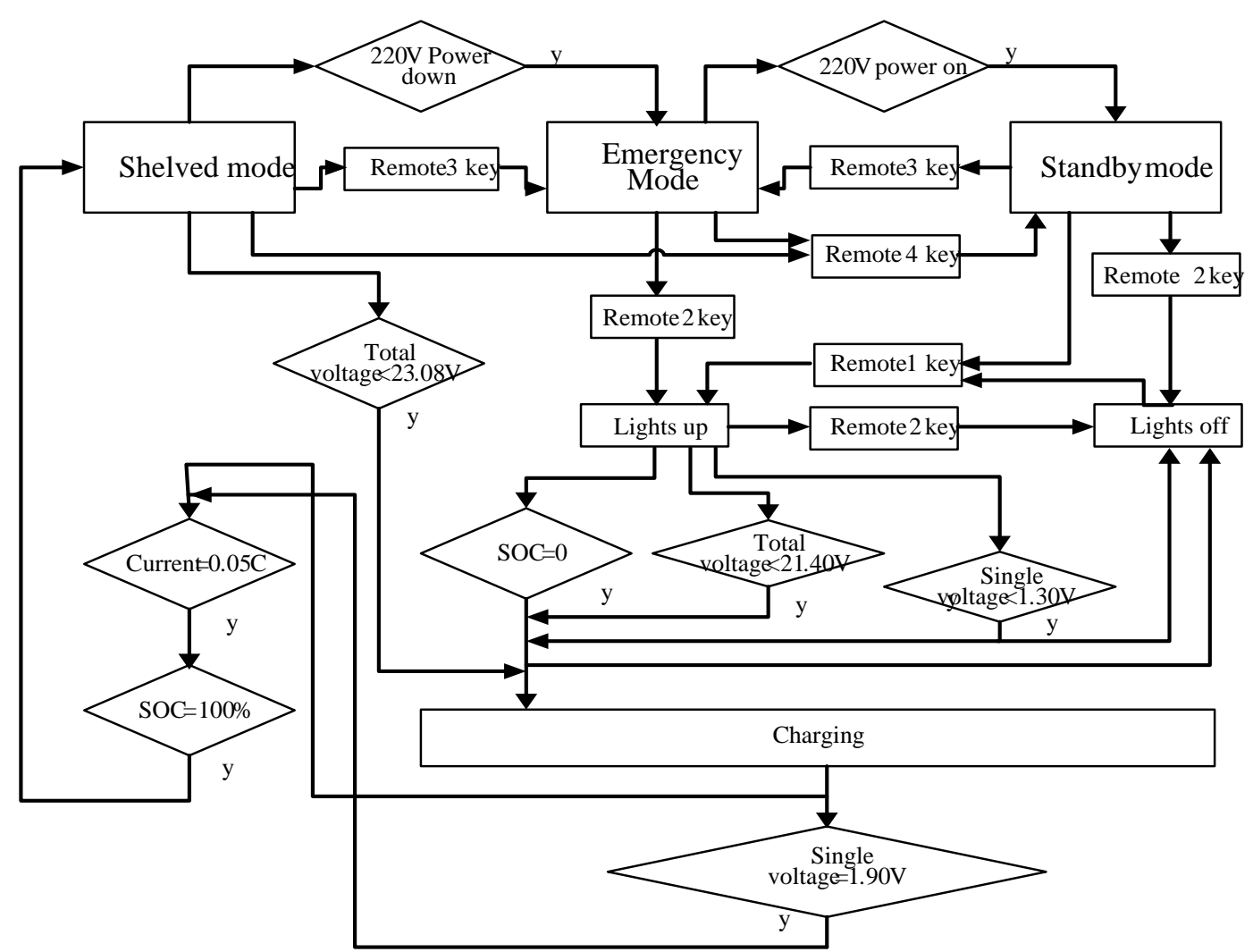

Figure 5. Emergency Lights Working Mode Transition Diagram

\subsection{Charge Management}

When the battery SOC is $0 \%$, according to the charging requirements $(0.5$ constant current charging $120 \mathrm{~min}$, cutoff voltage $1.900 \mathrm{~V} \pm 0.004 \mathrm{~V}$ charge $180 \mathrm{~min}$, cut-off current $0.05 \mathrm{C}$, or stop charging when SOC $\geq 105 \%$ ), the charging process is controlled..

When the battery shelved voltage $<24.500 \mathrm{~V}$, floated or supplemented the battery power, the charging process is: When you first use $0.5 \mathrm{C}$ constant current charging, the single battery voltage charge to $1.900 \pm 0.004 \mathrm{~V}$, transferred to constant voltage $1.900 \pm$ $0.004 \mathrm{~V}$ charge, during constant voltage charging, when the SOC reaches $105 \%$ or the charging current is smaller than $0.05 \mathrm{C}$, the charging is stopped. Alternating charging balanced mode, so that each battery will charge to $1.900 \pm 0.004 \mathrm{~V}$.

\subsection{Discharge Management}

When the power supply from the battery to the emergency lights, when detecting single discharge voltage $\leq$ discharge cut-off voltage (any one battery discharge voltage $\leq 1.300 \pm$ $0.004 \mathrm{~V}$ ), immediately and automatically stops the battery discharges, and the use of red lamp is flashing fast alarm.

Both 220VAC and the battery are stopped power supply the emergency lights, when the battery voltage reaches the discharge cutoff voltage $(1.300 \pm 0.004 \mathrm{~V})$, Battery management system should automatically stop working to avoid battery power 
consumption caused by excessive battery discharge point; When 220VAC power is restored, the battery management system should be able to resume normal work.

Charging and discharging control unit schematics are shown in Figure 6.

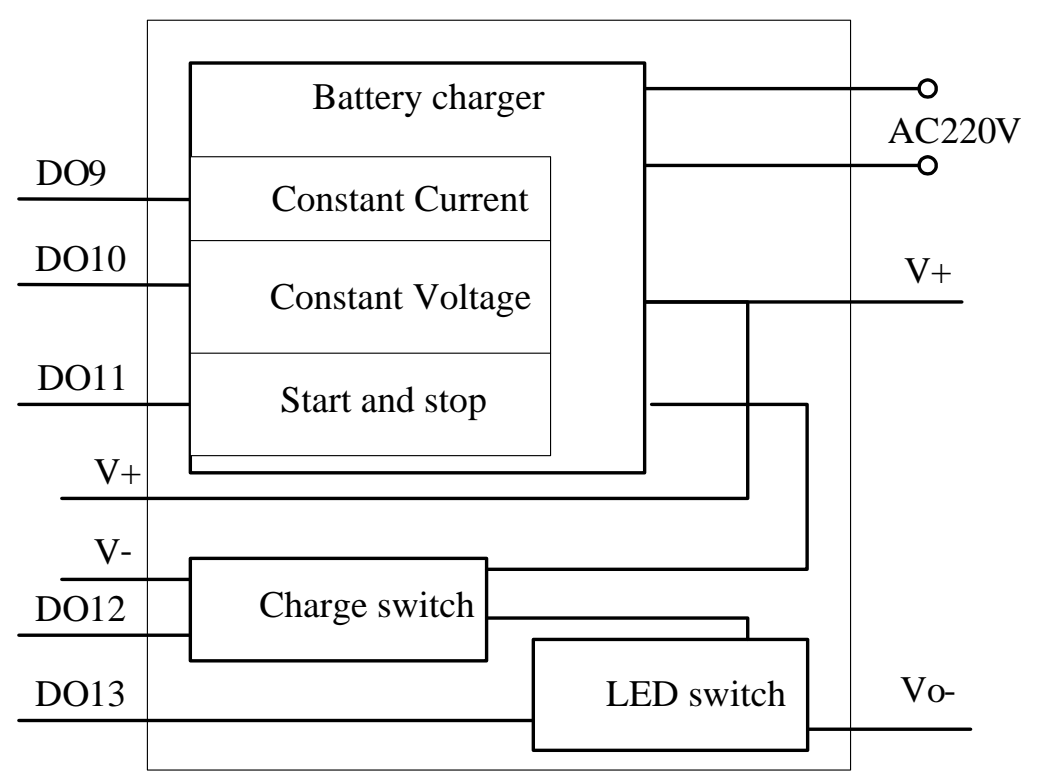

Figure 6. Charge and Discharge Control Unit Schematics

\section{System Software Design}

\subsection{Acquisition and Single Voltage Balancing}

The Sampling and balanced of Single voltage uses time-sharing and equilibrium method.

Sip1: turn on the single voltage sampling

Sip 11: cycle count I is set to 1 , empty single voltage buffer, the buffer address a is set to 1

Sip 12: closing the relay Jig, and is delayed, then single voltage is held on Can

Sip 13: Open relay Ji, after the delay, shut Jam, After further delay, start A / D , get Ji single battery voltage vi, and send into single voltage buffer.

Sip 14: loop counter $I+1$, buffer address a +1 , if the current loop counter $I$ is less than the number of the cells n, the Sip 1, otherwise Sip 15

Sip 15: End and the formation of single voltage v1-vn

Sip 2: the v1-vn of single voltage needs equalization calculation, to obtain the battery to be balanced, the unit cells to be balanced, the biggest single battery among v1-vn are considered to have balance need, then it is set to $\mathrm{Vb}$.

Sip 3: equilibrium for balanced single battery:

Sip 31: Close Vb corresponding single balanced circuit relays GJb and Jab relay

Sip 32: the charging current of GJb and Jab is bypassed and kept for some time

Sip 33: Open Jab and Gob

Sip 4: a

\subsection{Single Battery Control Methods of Charge Balance}

In order to play battery performance and extend battery life, balance control using the following strategies:

Suppose I as single battery serial number, the corresponding battery voltage is VI, introduction of energy management systems and single battery balanced. 
Equilibrium demand is calculated as follows, provided single voltage VI, where I is the sequence number corresponding to the single battery, the monomer is extracted equalization algorithm is as follows:

Sip1: Bati single battery voltage in turn collects VI, and into a linear form in LV;

Sip2: set up a temporary variable Vt, IMAX sending the first element and linear table $\mathrm{LV}$ [1] into the Vt, IMAX $=1$, set up a temporary variable index $=2$;

Sip 3: If the LV [index] > Vt, then Vt $=\mathrm{LV}$ [index], IMAX = index;

Sip 4: index +1 , if the index is greater than the number of single battery $N$, exit, return Imax value

Otherwise Sip 3.

\section{Implemented Measuring System Composition}

\subsection{Signal Detection}

(1) 14 single voltage signals, detection range is $0-2 \mathrm{~V}$, accuracy is $0.2 \%$;

(2) Total voltage signal is range: $0-30 \mathrm{~V}$, accuracy is $0.2 \%$;

(3) Current signal is ranges: $\pm 6 \mathrm{~A}$, accuracy is $0.5 \%$

(4) The detection signal $220 \mathrm{~V}$ AC of power supply 1 is a way for detecting power status.

\subsection{Control Signal}

The charger 2-way output is used to control constant voltage, constant current and stop work mode. There are two kinds of ways to control the emergency lights powered by a battery power source, and the other provides power from the charger.

Infrared remote control receiver for emergency light switch, the mode switching, self-testing and other control; Status lights with red, blue and green LED indicates the operating state of emergency lights, specific requirements, see the emergency lights work mode, the state of emergency lights and emergency lights display inspection function technical requirements.

\subsection{Power Supply}

When the battery is in standby $220 \mathrm{~V}$, power consumption is less than $0.5 \mathrm{~W}$.

\subsection{Ventilation Cooling}

Set a small exhaust fan, usually 220VAC power supply, power outage, powered by a battery pack. In the battery charging or emergency lights activated lighting (including the spare and emergency modes), exhaust fans to work.

\subsection{Charging Control}

Chargers should be made to meet the technical requirements of constant, constant voltage charging requirements; power is $180 \mathrm{~W}, 220 \mathrm{~V}$ AC power supply, the working environment temperature: $-30{ }^{\circ} \mathrm{C}-50{ }^{\circ} \mathrm{C}$.

Embodiment of the measuring system consists of:

1. Multiple sampling and balanced relay Gig, sampling control relay Jam and balanced relay Jab are served as dual relays MOS (Model: PS7113), The relay on-resistance is $0.9 \Omega$, maximum current is $300 \mathrm{~mA}$, according to the single voltage of different types battery, by selecting different current limiting resistor, the equilibrium current is limited within the allowable range, in this instance the battery is nickel-zinc batteries, single nominal voltage of $1.72 \mathrm{~V}$, the current limiting resistor $\mathrm{R} 0, \mathrm{R} 1, \ldots \ldots . \mathrm{Rn}$ design value of $1.4 \Omega$. 
2. Cg is the capacitor of sample and hold, the capacitor is $0.1 \mathrm{uF}$.

3. System power switch (Jig) uses STANSON STN607D P-channel MOS transistor The gate electrode drive circuit of PMOS transistor is use of PC817 opt coupler GJctr1 and GJctr2 constituted for the completion of the exchange device power-up and power self-down control; AC power input interface chip GJfback is adopted SHARP PC817 opt coupler for the completion of $\mathrm{AC}$ power status detection and signal isolation [15].

4. Battery charge and discharge control switches (GJchar) and the power switch of LED lamp are adopted Jed STANSON STN4110 N-channel MOS transistor, NMOS gate electrode is controlled by PC817 photo coupler driven. Emergency light charging voltage curve is shown in FIGURE 5.

5. CPU adopted C8051F410 with a 12 ADC interface and multi-channel digital IO interface, to complete the $\mathrm{AD}$ conversion by $\mathrm{ADC}$, to complete all MOS relay switch controlled by the digital IO port and decoder, Specific measurement, control and data processing calculations done by the software.

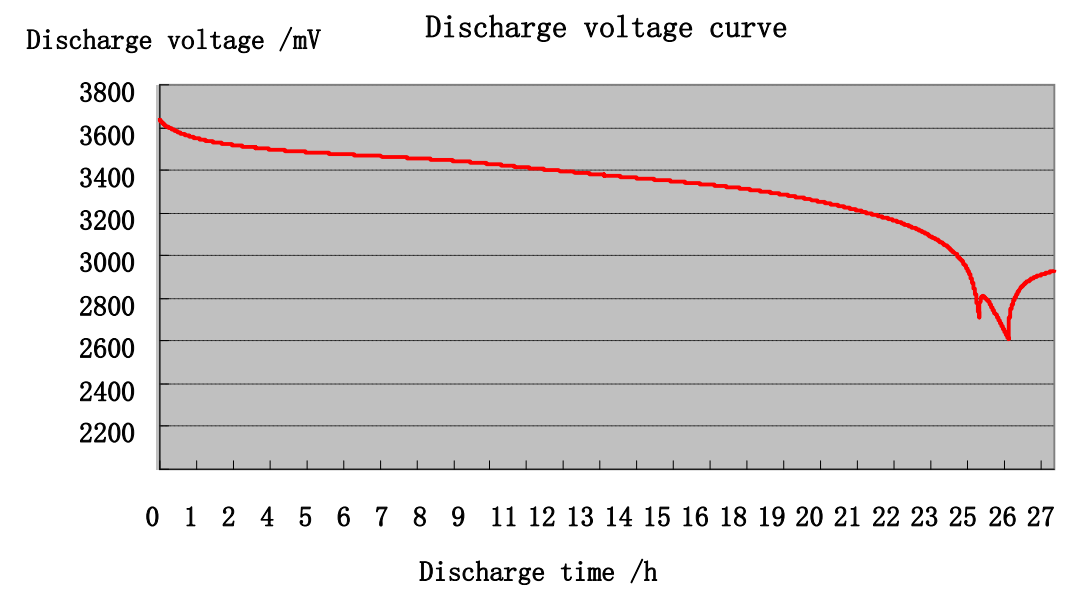

\section{Figure 7. Emergency Light Charging Voltage Curve}

\section{Conclusion}

Develop monitoring and balancing of battery, charge and discharge protection of battery, multi-mode switch, LED emergency light battery management control device and method for remote control and other features, to improve the safety and reliability of emergency lights, to extend emergency lights life and enhance operability and extended scope of application of the emergency lights have an important role.

\section{References}

[1] S. X. Wan, L. Ting and L J. Gu Liang, "Rocket propulsion", vol. 37, no. 2, (2011).

[2] X. Zhai and J. Wang, "Realize infrared remote control in embedded systems", J. Development and application", vol. 6, no. 5, (2010), pp. 24-26.

[3] J. Lu, "J. Control Technology", vol. 32, no. 1, (2013), pp. 23-32.

[4] M. Rico-Secades, "IEEE Transactions", vol. 41, no. 5, (2005), pp. 1386-1390.

[5] T.-C. Wang, Z. Bo and Q. Wang, "J. Harbin Institute of Technology Journal”, vol. 40, no. 9, (2008), pp. 1421-1425.

[6] S. Wang, T.-T. Liu and G. Li, "reliable detection adopted finite state machine control instructions [J]", rocket propulsion, vol. 37, no. 5, (2011), pp. 63-68.

[7] L. Yun Hai, "J. Refining and Chemical Industry", vol. 21, no. 3, (2010).

[8] Y. Feng, H. Wang and X. Liang, "J .Control Technology", vol. 29, no. 9, (2010), pp. 54-57.

[9] L. B. Yang, W. Xu and S. Liu, "J. Wuhan University of Technology", vol. 34, no. 6, (2012), p. 712.

[10] J.-c. Jiang, F. Wen and J.-p. Wen, "J. Power Electronics", vol. 45, no. 12, (2011).

[11] Y. Feng, "J. Control Technology", vol. 29, no. 9, (2010), pp. 54-57.

[12] Y. Chunlei, “J. Shanghai Jiaotong University”, vol. 45, no. 8, (2011), pp. 1186-1190. 
[13] X. Zheng, Y. Limei and F. Sun, "Shanghai Motor College", vol. 15, no. 3, (2012), pp. 152-157.

[14] L. Bingyang, X. Wenjing, L. Sining, M. Yimin, "Wuhan University of Technology", vol. 34, no. 6, (2012), pp. 712-715.

[15] N. Lotfi and P. Fajri, "J. Energies", vol. 6, (2013). 
International Journal of Control and Automation Vol.8, No.5 (2015) 